\title{
FORCE MODULATION AS A SOURCE OF HAND DIFFERENCES IN RAPID FINGER TAPPING *
}

\author{
John I. TODOR and Ann L. SMILEY-OYEN \\ University of Michigan, Ann Arbor, USA
}

Accepted August 1986

\begin{abstract}
The purpose of this study was to examine the factors contributing to hand differences in rapid single finger tapping. To this end, task-defined temporal variables and motor outflow, as reflected by the magnitude and duration of force, were simultaneously measured. Thirty-one right-handed college age subjects performed a rapid finger-tapping task with the index finger of the right and left hands. The order of hand use was counterbalanced across subjects. Significant differences favoring the right hand were found in the rate of tapping as indicated by the inter-tap interval, dwell (duration of key closure), the interval between force peaks and the variance of the force peak intervals. Additionally, relative to the left hand, the right hand used significantly smaller amounts of force and exhibited less variation in force. These data are conceptually consistent with Kimura's (1979) contention that the left hemisphere is uniquely specialized to control sequences of action such as postural transitions. However, Kimura argues that this unique ability is manifest in multiple-finger sequences but not rapid single finger tapping. The present data indicate such a mechanism may be observed in rapid single finger tapping if the appropriate dependent measures are obtained.
\end{abstract}

\section{Introduction}

Amongst right-handed individuals a common, and in fact, expected observation is for the right hand to have a more rapid single finger tapping rate than the left (Reitan and Davidson 1974). However, the basis of this rate difference has not been clearly delineated. Both Peters (1980) and Todor and Kyprie (1980) found faster right hand finger tapping to occur in the phase of movement where directional reversals were made. Similar to Kimura $(1977,1979)$, the investigators of both studies inferred that the hands differed in their ability to make postural

* Requests for reprints should be sent to A.L. Smiley-Oyen, Dept. of Kinesiology, University of Michigan, Ann Arbor, MI 48109, USA. 
transitions. These studies. however, do not directly support this contention, nor do they provide insight into the controlled movement variable that would give rise to a hand difference in this phase of movement.

The present study examined the extent to which the ability to control directional reversals accounts for hand differences in rapid single finger tapping. To this end, task defined temporal variables and motor outflow, as reflected by the magnitude and duration of force in the upward and downward direction, were simultaneously measured. Since the force variables more directly reflect the activity of the motor system, one should be able to determine if hand differences in the rate of tapping reflect qualitative differences in the pattern of movement control.

\section{Method}

Subjects

Thirty-one college age subjects participated in this study (16 females, 15 males). Each subject wrote with his/her right hand.

\section{Apparatus}

The apparatus consisted of a modified standard telegraph key. Strain gauges were mounted on the shaft of the key to measure the force applied. The diameter of the key head was reduced to $1.90 \mathrm{~cm}$ to decrease the variability in finger positioning. Velcro straps attached to the underside of the key were used to secure the finger to the key, further restricting the position of the finger. Strapping the finger to the key enabled force measurements to be made in an upward direction, i.e., above the normal resting position, as well as force in the downward position. To make key contact from the resting position a downward finger movement of $3 \mathrm{~mm}$ was required. Arm rests provided support for the forearms. Velcro straps were used to secure the forearms to the armrests, thus restricting movement of the elbow and shoulder joints. To eliminate movement at the wrist, the uninvolved fingers and thumb were positioned in contact with a flat surface adjacent to the telegraph key. Further, by positioning the hand to require extension of the index finger to reach the key, movement was restricted to flexion and extension of the metacarpal phalangel joint.

Key closure completed a 3 volt DC circuit thus enabling a distinction to be made between the dwell or key closure phase $(+3$ volts) and the slack or key open phase $(0$ volts) of each tap. This circuit, signaling key position, and the amplified output of the strain gauges were fed into independent channels of a computer.

On-line computer analysis of both channels was performed continuously throughout each trial. The first tap was eliminated from the analysis to reduce the impact of 


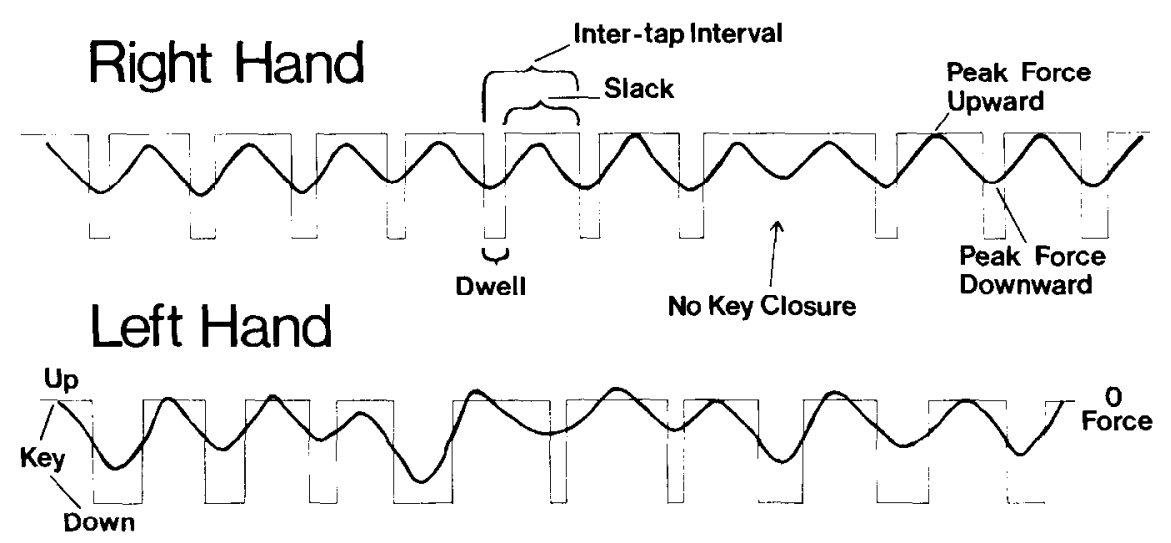

Fig. 1. Sample performance profile of the right and left hands.

reacting to the starting tone. The two signals were simultaneously sampled every one msec to produce the bases of computing the following parameters (see fig. 1):

- Inter-tap Interval: the time between the onset of successive key closures.

- Dwell: the length of time the key was in the down position (i.e., the duration of a key closure).

- Slack: the time between successive dwell periods (i.e., when the key was not in the down position).

- Peak Force Downward: the maximum force value attained during a dwell period, expressed in grams.

- Peak Force Upward: the minimum positive force or the maximum negative force value attained during a slack period. In the normal resting position of the key the strain gauges signaled zero force, thus negative force values indicated the extent to which the key was pulled upward by the finger during the slack period.

- Peak Force Interval: the time between successive force peaks in a downward direction.

- Failed Key Closures: the number of times/trials that a downward force peak was detected that was not accompanied by a key closure, i.e., force was insufficient to produce key contact.

- Failed Key Openings: the number of times/trials that two downward force peaks were observed during a single dwell period, i.e., the drop in force between force peaks was insufficient to break key contact.

\section{Procedures}

Subjects performed three consecutive trials of rapid finger tapping with each hand. They were instructed to tap continuously as rapidly as possible between the auditory signals indicating the beginning and end of a trial. Trials lasted 8 seconds and were 
separated by a 30-second interval. The order of hand use was counterbalanced across subjects and gender. Subjects were not given any information about their performance. They were, however, able to hear key contacts during tapping and their hands were in full view throughout testing.

\section{Results}

The mean of the three trials was computed for each hand for all parameters measured. Each of these values and their corresponding within-subject variance were subjected to a repeated measure analysis of variance (hand $\times \operatorname{sex} \times$ order). Although certain order effects were significant, they did not impact directly on the current issues or interpretation. These order effects are discussed at length elsewhere (Todor and Smiley-Oyen 1986).

The right hand was significantly faster than the left hand on the following rate variables: the inter-tap interval $(F(1,27)=9.98, p<0.004)$, dwell $(F(1,27)-26.42$, $p<0.0001)$, and the interval between downward force peaks $(F(1,27)=19.62, p<$ 0.0001 ). The hands did not differ significantly in the duration of the slack period (see fig. 2). There were no sex differences or hand by sex interactions for any of the rate variables.

The within-subject variance of the interval between downward force peaks was significantly smaller for the right hand $(F(1,27)=13.44, p<0.001)$. On this parameter,
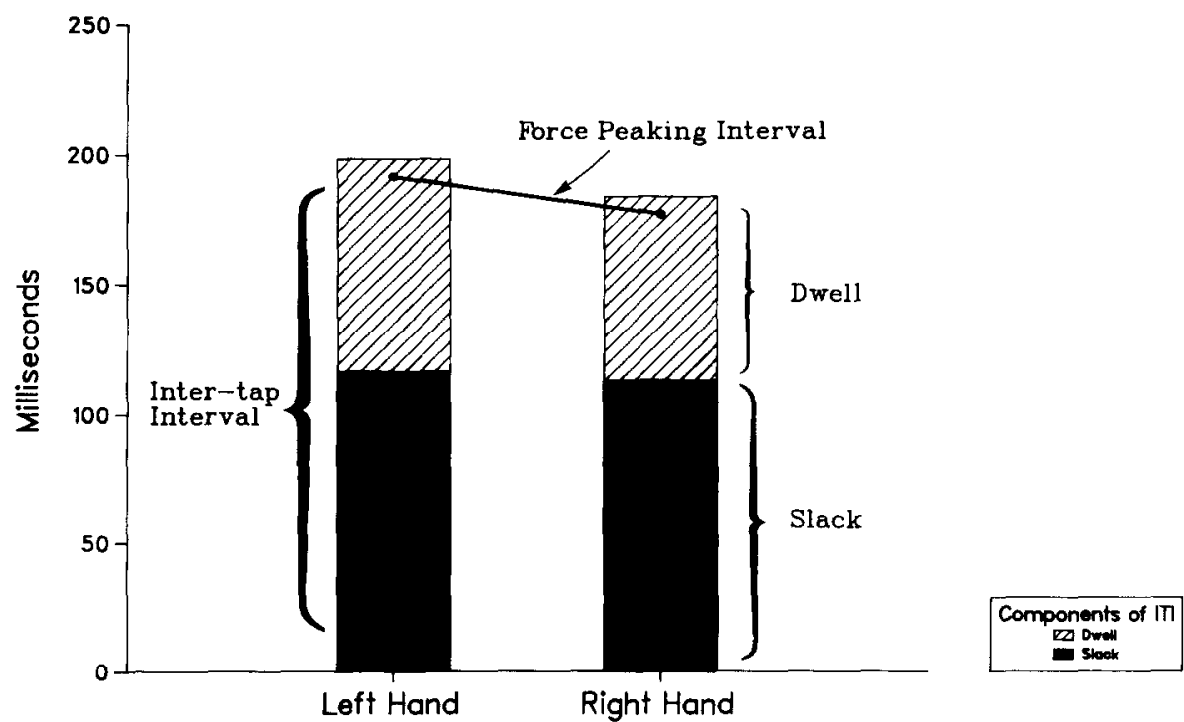

Fig. 2. Mean values for rate variables by hand. 


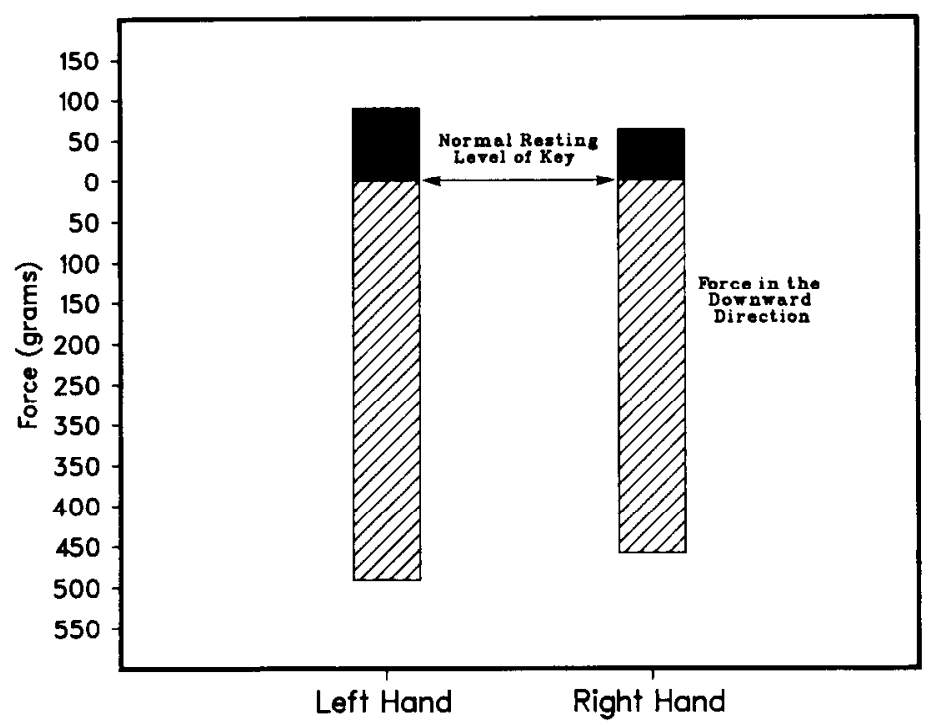

Direction of Force Lopwerd Fores

Fig. 3. Range of force in rapid finger tapping by hand.

females were less variable than males $(F(1,27)=4.65, p<0.04)$. No other hand or sex differences in the variance of rate variables were significant.

No hand or sex differences in peak force downward or upward reached the conventional $p<0.05$ level of significance. However, greater average downward force by the left hand was marginally significant $(p<0.065)$. The range of force (peak force downward-peak force upward) per tap was significantly lower for the right hand $(F(1,27)=4.222, p<0.05)$ (see fig. 3). Right hand variance was less than the left hand for both peak force downward $(F(1,27)=12.63, p<0.001)$ and peak force upward $(F(1,27)=4.17, p<0.05)$. Additionally, females exhibited significantly less variance on both measures $(F(1,27)=5.28, p<0.03)$ and $(F(1,27)=3.93, p<0.05)$ for peak force downward and upward, respectively.

At least one failed key closure or failed key opening occurred on $77.4 \%$ of all trials. Trials with at least one failed key closure (RH $-72 \%, \mathrm{LH}-76 \%$ of trials) occurred more frequently than those with failed key openings ( $\mathrm{RH}-10.8 \%, \mathrm{LH}-22.6 \%$ of trials). A non-parametric pairwise comparison between failed key closures with the right and left hand, summed across the three trials, was not significant. Since only 13 of the 31 subjects exhibited a failed key opening with either hand, no statistical comparisons were made.

\section{Discussion}

This investigation supports the argument made by Todor and Kyprie (1980) and Peters (1980) that the efficiency in executing postural 
transitions or directional reversals can account for hand differences in rapid single finger tapping. This position is conceptually consistent with Kimura's view of the left hemisphere as having a unique role in guiding postural transitions. It is, however, in contrast to Kimura's contention that this left hemispheric function affects only complex or multi-finger sequences. It is argued here that when appropriately measured, it can be observed to operate in rapid single finger tapping as well.

Consistent with previous reports the superior rate of tapping exhibited by the right hand was attributable to a shorter duration of the key depression (Peters 1980; Todor and Kyprie 1980). Both previous studies argued that the hand differences in single finger tapping are selectively associated with this variable because it reflects the process of reversing the direction of movement. In contrast, the slack time, as measured in this study, included the time necessary to traverse the distance from up-to-down and down-to-up. Peters (1980) clearly demonstrated that the hands did not differ on this component of tapping. One could argue that the slack time also included a directional reversal (up-to-down) and therefore should reflect hand differences. The fact that there were no hand differences in this component is likely due to two factors: (1) the measurement of the postural transition was confounded by the inclusion of the distance covering component, and (2) the instruction "to "tap" as fast as possible' coupled with the fact that a physical key closure or tap occurred only in the downward direction.

In addition to slower dwell times, the left hand also had a longer average time between successive force peaks (i.e., key being pushed downwards). Thus, the left hand was not only slower in completing the directional reversals but also was typically slower in generating the motor outflow necessary for successive taps. It is, of course, likely that longer dwell durations influence the delay between force peaks.

As previously reported, there was evidence that the left hand was more variable in its tapping rate. However, while hand differences in the variance of the inter-tap interval and dwell have been previously reported (Peters 1980; Peters and Durding 1978; Todor and Kyprie 1980), that was not the case in this study. Rather, of the rate variables, only the variance in the interval between downward force peaks was significant. This apparent discrepancy can be attributed to the detection and elimination of failed key closures/openings from the computation of both the inter-tap interval and dwell in the present study. 
Thus, in previous studies, greater left hand variance in these parameters could have been associated with lateralized differences in the frequency and/or duration of failed key closures/openings, both of which would have increased the observed variance.

Previously, Todor and Kyprie (1980) indicated that subjectively the non-preferred hand seemed more variable in its regulation of force. Similarly, Peters (1980) inferred that force modulation was a key factor in accounting for hand differences in single finger tapping. In his experiment, hand differences became more pronounced when the range of motion was reduced. According to Peters, this occurred because greater demands were placed on the ability to precisely time the activation of flexor and extensor muscles which resulted in force modulation. The results of the present study confirmed and extended these previous inferences. In this study, the force exerted was continuously monitored. On the average, the range of force exerted in a downward and upward direction was greater in the left hand. Assuming the rate of rise in force did not differ substantially between the hands, one would expect the additional time spent in exerting force within a tap to contribute to the observed rate differences.

As is strikingly evident in fig. 1, the force exerted by the left hand was more variable in both directions. That is, not only did the left hand exert variable amounts of force during key depression but it also varied more in the amount of force applied in an upward direction. While both hands occasionally modulated a transition from extension to flexion before reaching the resting level of the key, they also occasionally pulled the key upwards beyond its normal resting position. These occurrences were much more pronounced and frequent in the left hand.

As mentioned above, the impact of failed key closures/openings on the measurement of rate variables could be substantial. Considering the frequency with which these phenomena were observed (at least one occurrence on $77.4 \%$ of all trials), it is essential that they be considered when evaluating movement planning. Both events represent a failure to comply with the external task demands. That is, the motor act was either inappropriately planned or the motor plan was ineffectively executed.

No lateral differences were observed in these phenomena. However, based on the fact that the right hand had a smaller average range of force and evaluation of the individual tracings, it is conceivable that failed key closures/openings occurred for different reasons in the two 
hands. The right hand appeared to minimize the force needed to complete the key closure and opening thereby increasing the probability of exerting insufficient force. In contrast, the left hand tended to exert more force and was more variable in its range of force. Rather than producing a failed key closure/opening because it minimized the force needed, the left hand appeared to be less capable of rapidly and consistently carrying out the force modulation because it failed to or was more variable in setting a point around which to begin directional reversals. That is, the movement planning underlying the left hand's tapping less effectively complies with or accommodates to the task demands.

Independent of whether lateral differences occur in failed key closures/openings, monitoring these phenomena could be useful when studying movement planning disorders. For example, both Roy (1981) and Kimura (1977) found that in a movement sequencing task, patients with left hemispheric damage exhibited frequent perseverations. A potentially informative extension of these studies would be to determine if perseverations involved ineffective efforts to change the movement or a failure to appropriately generate a change in the motor plan.

In summary, in rapid single finger tapping, hand differences are greatest in the phase of the tap cycle requiring postural transitions. The left hand appears to be less capable of rapidly carrying out this process because it is more variable in setting a point around which to begin directional reversals. Thus, it seems reasonable to conclude that the control of left hand tapping is not simply a slower version of the right, and may, in fact, be qualitatively different.

\section{References}

Kimura, D., 1977. Acquisition of a motor skill after left hemisphere damage. Brain 100, 527-542.

Kimura, D., 1979. 'Neuromotor mechanisms in the evolution of human communication'. In: H.D. Steklis and M.J. Raleigh (eds.), Neurobiology of social communication in primates: an evolutionary perspective. New York: Academic Press.

Peters, M., 1980. Why the preferred hand taps more quickly than the non-preferred hand: three experiments on handedness. Canadian Journal of Psychology 34, 62-71.

Peters, M. and B.M. Durding, 1978. Handedness measured by finger tapping: a continuous variable. Canadian Journal of Psychology 32, 257-261.

Reitan, R. and L. Davidson, 1974. Clinical neuropsychology: current status and applications. Washington, DC: V.H. Winston and Sons. 
Roy, E.A., 1981. 'Action sequencing and lateralized damage: evidence for asymmetries in control'. In: J. Long and A. Baddeley (eds.), Attention and performance IX. Hillsdale, NJ: Erlbaum.

Todor, J.I. and P.M. Kyprie, 1980. Hand differences in the rate and variability of rapid tapping. Journal of Motor Behavior 12, 57-60.

Todor, J.I. and A.L. Smiley-Oyen, 1986. The influence of order of hand use on lateral asymmetries in rapid finger tapping. Manuscript submitted for publication. 